\title{
Generalized and Mucosal Melanosis Associated with Ultra-Late Recurrence of Malignant Melanoma
}

We describe a patient with progressive hyperpigmentation of the skin and gastrointestinal melanosis. The pigmented lesions in the stomach and duodenum served as a diagnostic sign of recurrent disease, 26 years after excision of melanoma of the skin.

A barium swallow and gastroduodenoscopy were performed in a healthy 63year-old man who had been suffering from vague abdominal symptoms for a few months. Multiple flat and polypoid pigmented lesions, varying from brown to black, were observed in the stomach and duodenum (Figures 1, 2). A malignant melanoma had been excised from his neck 26 years previously. The new biopsies from the pigmented lesions in the upper gastrointestinal tract showed only melanotic pigment within macrophages and no malignant cells were found. Staining with S-100 and HMB-45 was negative. On examination, no suspected pigmented nevi were detected on the skin or mucous membranes. Normal fundoscopy and rectoscopy findings ruled out the possibility of intraocular or anal melanoma.

The results of a computed tomography scan showed diffuse liver metastasis, and some thickening of the gastric wall at the fundic area. A liver biopsy confirmed the diagnosis of metastatic malignant melanoma. At 2 months later the patient no-

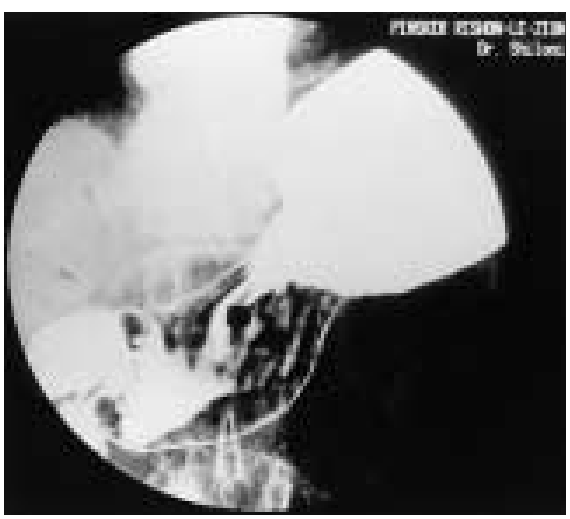

Figure 1 The typical "bullseye" in the gastric wall ticed darkening of the skin, especially in exposed areas, and voided dark urine. On examination, the skin and mucous membranes were slate gray, especially on the face, neck, chest and upper extremities.

The patient died from the disease 7 months after the initial diagnosis.

Malignant melanoma is the most common malignancy to metastasize to the gastrointestinal tract, involving the small intestine, colon, stomach and esophagus [1-3]. The typical endoscopic finding is the "bullseye" or "target" lesions [1,2]. As far as we know, this is the first described case of gastrointestinal melanosis associated with melanosis cutis, appearing in a patient with ultra-late recurrence of the disease $[4,5]$.

\section{T. Friedman ${ }^{1}$, M. Friedman ${ }^{2}$, \\ R. Weitzen ${ }^{3}$, E. Scapa ${ }^{2}$}

${ }^{1}$ Department of Plastic Surgery,

Assaf Harofeh Medical Center,

Affiliated to Sackler Faculty of Medicine,

Tel Aviv University, Israel

2 The Institute of Gastroenterology,

Liver Diseases and Nutrition,

Tel Aviv University, Israel

${ }^{3}$ Department of Oncology,

Tel Hashomer Medical Center,

Affiliated to Sackler Faculty of Medicine, Tel Aviv University, Israel

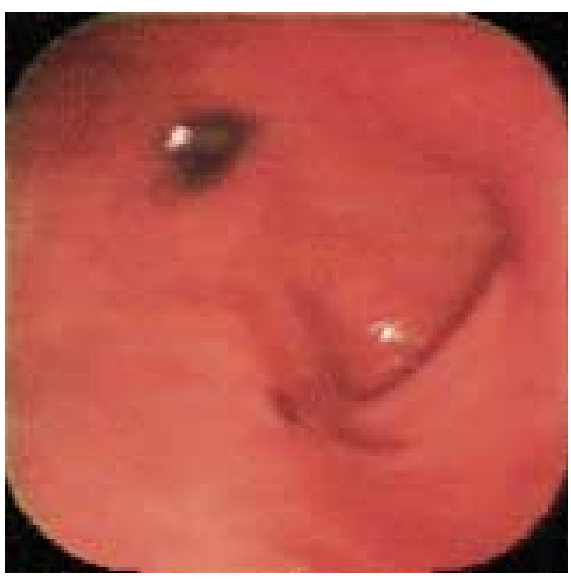

Figure 2 Brownish appearance of gastric lesion

\section{References}

${ }^{1}$ Reintgen DS, Thompson W, Garbutt J. Radiologic, endoscopic and surgical considerations of melanoma metastatic to the gastrointestinal tract: endoscopic experience. Surgery 1984; 95: 635-639

${ }^{2}$ Kadakia SC, Parker A, Canales L. Metastatic tumors of the upper gastrointestinal tract: endoscopic experience. Am J Gastroenterol 1992; 87: 1418-1423

${ }^{3}$ Horowitz M, Nogrega M. Primary anal melanoma associated with melanosis of the upper gastrointestinal tract. Endoscopy 1998; 30: 662-665

${ }^{4}$ Sohn N, Gang H, Gumport SL et al. Generalized melanosis secondary to malignant melanoma. Cancer 1969; 24: 897-903

${ }^{5}$ Holcomb BW, Thigpen JT, Puckett JF, Morrison FS. Generalized melanosis complicating disseminated malignant melanoma in pregnancy: a case report. Cancer 1975; 35: 1459-1464

\section{Corresponding Author}

\section{E. Scapa, M.D.}

Institute of Gastroenterology

Liver Diseases and Nutrition

Assaf Harofeh Medical Center

Zerifin 70300

Israel

Fax: $\quad$ +972-8-9779727

E-mail: escapa@asaf.health.gov.il 